\title{
Basis set extrapolations for density functional theory
}

\author{
Peter Kraus* \\ School of Molecular and Life Sciences, \\ Curtin University, \\ GPO Box U1987, Perth 6845, WA, Australia
}

\section{${ }_{13} 1$ Introduction}

\begin{abstract}
Basis set extrapolation is a common technique in wavefunction theory, used to squeeze better performance out of the highest affordable level of theory by combining it with lower quality calculations. In this work, I present analogous techniques for basis set extrapolation in density functional theory, focusing on $[2,3]-\zeta$ calculations, and including double hybrid and dispersion corrected functionals. My recommendations are based on basis set limit data from finite element calculations, estimates of basis set limits for double hybrid corrections, and they are validated using the GMTKN55 and NCDT datasets. A short review of extrapolation methods for Hartree-Fock calculations based on modern finite element data is carried out to inform this work. Extrapolation of $[2,3]-\zeta$ calculations in cc-pv $X z$-pp and def2- $X$ vpd basis sets with the proposed recipes routinely matches and sometimes outperforms $4-\zeta$ calculations at a fraction of the cost. The methods are implemented in Psi4, allowing for an automated and intuitive application.
\end{abstract}

The concept of estimating complete basis set (CBS) energies using basis set extrapolation methods is nothing new for practitioners of wavefunction theory (WFT). Usually, such extrapolation methods involve calculating absolute energies using correlation-consistent basis sets of consecutive $\zeta$-qualities, with Helgaker's power formula and cubic scaling (Eq. (1)) being one common example. ${ }^{[1]}$

*E-Mail: peter.kraus@curtin.edu.au 


$$
E_{\infty}^{\mathrm{corr}}=E_{X}^{\mathrm{corr}}-A X^{-\alpha}, \alpha=3
$$

Here, $E_{\infty}^{\text {corr }}$ is the correlation (i.e. post-Hartree-Fock) energy at an infinite basis set size, while $X$ denotes the cardinal number of the finite basis set, with this number usually equivalent to the $\zeta$-ness of the basis set. The parameter $A$ is system-dependent and is always fitted to $E_{X}^{\text {corr }}$ data. Many different variants of Eq. (1) have been proposed since, sometimes replacing the power scaling $A X^{-\alpha}$ term with exponential $\left(A e^{-\alpha X}\right),{ }^{[2]}$ exponential-square-root $\left(A e^{-\alpha \sqrt{X}}\right.$, expsqrt in the following) or other functions. Results of such $[X-1, X]-\zeta$ extrapolations are usually comparable to the results from a $X+1-\zeta$ calculation, provided sufficiently large basis sets are used $(X>=4) .{ }^{[3]}$ Smaller basis sets might perform better with a specifically tailored $\alpha \cdot{ }^{[4]}$

The Hartree-Fock (H-F) component of WFT calculations converges to the basis set limit rather quickly when compared to the correlation energy. In Truhlar's H-F extrapolation scheme, derived from estimates of Ne, HF, and $\mathrm{H}_{2} \mathrm{O}$ basis set limits, Eq. (1) is applied with $\alpha=3.4$ which is optimal for a [2,3]- $\zeta$ extrapolation. ${ }^{[4]}$ Halkier et al. have proposed the use of Eq. (2) for extrapolating Hartree-Fock energies:

$$
E_{\infty}^{\mathrm{H}-\mathrm{F}}=E_{X}^{\mathrm{H}-\mathrm{F}}-A e^{-\alpha X}, \alpha=1.63
$$

This "global" $\alpha$ parameter has been determined from fits of [2-6]-, [3-6]-, and [2-5]- $\zeta$ energies of diatomic molecules using correlation-consistent basis sets, with $E_{\infty}^{\mathrm{H}-\mathrm{F}}$ values sourced from numerical H-F results of 9 species available at the time. ${ }^{[5]}$ This extrapolation method performs well, and it is the default H-F extrapolation method in Psi4. ${ }^{[6]}$ A three-point variant of Eq. (2), where both $\alpha$ and $A$ are fitted to results from $[X-2, X-1, X]-\zeta$ calculations, is used in CFOUR, ${ }^{[7]}$ despite the lower generality of this variant. ${ }^{[5]}$ The exponential-square-root (expsqrt) function, shown in Eq. (3), is used by default in ORCA, 


$$
E_{\infty}^{\mathrm{H}-\mathrm{F}}=E_{X}^{\mathrm{H}-\mathrm{F}}-A e^{-\alpha \sqrt{X}}
$$

with values of $\alpha$ depending on basis set family as well as size. ${ }^{[8]}$ Many other extrapolation schemes are available, for a more detailed overview see the recent review of Varandas. ${ }^{[9]}$

In density functional theory (DFT), extrapolation methods have gained little traction. Raymond and Wheeler reported that while a [3,4]- $\zeta$ extrapolation applied to a hybrid density functional approximation (DFA) produces reasonable values, the improvements over $4-\zeta$ results are negligible. ${ }^{[10]}$ Jensen showed that three-point extrapolation schemes (i.e. where both $A$ and $\alpha$ are fitted) applied to a semi-local DFA do not perform well when contracted polarisation-consistent basis sets are used, and two-point schemes perform similarly to the non-extrapolated results. ${ }^{[11]}$ This is attributed to the fast convergence of DFT with respect to basis set size, which is comparable to H-F, and is therefore much quicker than for correlated WFT methods. ${ }^{[11,12]}$ Furthermore, the fitting of functional parameters during the development of DFAs may correct for basis set incompleteness, ${ }^{[11]}$ which is why it's recommended to use basis sets comparable to the ones used during the development of such methods. ${ }^{[13]}$

With the advent of double hybrid DFAs, which include a correlation component based on secondorder Møller-Plesset perturbation theory (MP2), the outlook for basis set extrapolation might have changed. Similarly, various treatments of dispersion forces have different dependencies on basis set sizes. To the best of my knowledge, basis set extrapolation results have been published to-date for only one double hybrid DFA, with a focus on chemical kinetics. ${ }^{[14,15]}$ The changes in the density functional zoo therefore warrant another visit to the extrapolation pavillion. The goals of this work are the following: i) revisit extrapolation schemes for H-F, obtaining values of $\alpha$ derived from modern numerical H-F data; ii) investigate basis set convergence for double-hybrid and dispersion-corrected density functionals as such, i.e. would an extrapolation work and does it need to be different for single and double hybrids; iii) propose, implement, and validate a reasonable and transferrable extrapolation scheme for DFAs; and iv) gauge whether such methods are worth it, i.e. whether a $[2,3]-\zeta$ extrapolation can approach $4-\zeta$ results. I have decided to focus on $[2,3]-\zeta$ extrapolations, as performance of DFAs does not seem to improve significantly past $4-\zeta$, and there are many large systems for which DFT applied with $3-\zeta$ basis 
sets is the currently highest affordable level of theory.

\section{Computational methods}

In this study I investigated the convergence behaviour of three families of basis sets: i) the correlationconsistent "Dunning" series supplemented by effective core potentials (ECP), cc-pv $X \mathrm{z}-\mathrm{pp}(X \in[\mathrm{d}, \mathrm{t}, \mathrm{q}, 5])$; ii) the polarisation-consistent contracted and segmented variant of the "Jensen" sets, pcseg- $N(N \in$ $[0,1,2,3,4]$, with $X \approx N+1)$; and iii) the "Karlsruhe" family of basis sets, including their ECPs, in (singly-)polarised and augmented variants, def2- $X$ zvp and def2- $X$ zvpd, respectively $(X \in[\mathrm{s}, \mathrm{t}, \mathrm{q}])$. I have included the Dunning basis sets for comparison with previous works, as well as due to their widespread use in DFT calculations. However, some recent works showed that more modern basis sets constructed with DFT in mind can be superior to the original Dunning sets for DFT applications. ${ }^{[11,16,17]}$ The Jensen basis sets were included as they are modern, and 5 levels of quality are available. Unfortunately, Jensen basis sets for heavy atoms are not available. Finally, the two variants of the Karlsruhe basis sets were included as several functionals have been parametrised using these basis sets, and augmented variants are available across the whole periodic table. Their main downside compared to the Dunning and Jensen basis sets is the lack of a $5-\zeta$ variant.

To obtain estimates of basis set limits, the H-F energies of two databases of diatomic molecules, and MP2 energies of the 15 reference species were calculated using Psi4 1.4a2.dev532. ${ }^{[6]}$ The direct algorithm for the evaluation of the electron repulsion integrals was used throughout. For all DFT calculations a $(150,974)$ quadrature grid was used. The MP2 and DFT basis set limits were estimated from calculations in $[3,4,5] \mathrm{ZaPa}-\mathrm{NR}-\mathrm{CV}$ basis sets. ${ }^{[18]}$ All MP2 and double-hybrid DFA calculations were carried out with core electrons frozen, including the $4 \mathrm{f}$ shell for post-lanthanides. Tightened convergence criteria for the density and energy $\left(<10^{-10} \mathrm{Eh}\right)$ were applied in all of the above calculations.

Sampling the large collection of available DFAs and basis sets is always going to introduce biases: an exhaustive study is not feasible. For a list of the DFAs that were selected, see Table 1. I have included the functionals BLYP-D3(BJ), PBE-D3(BJ), B3LYP-D3(BJ), and B2PLYP-D3(BJ) for their popularity in their respective classes. The remaining two generalised gradient approximation (GGA) functionals, revPBE-D3(BJ) and B97-D3(BJ), are good performers in the GMTKN55 benchmark, ${ }^{[19]}$ which war- 
Table 1: Overview of studied DFAs. References correspond to functionals and parametrizations of dispersion corrections.

\begin{tabular}{l|l|l|l}
\hline Method name & DFA type & Disp. type & Reference \\
\hline BLYP-D3(BJ) & GGA & D3(BJ) & {$[20-22]$} \\
PBE-D3(BJ) & GGA & D3(BJ) & {$[22,23]$} \\
revPBE-D3(BJ) & GGA & D3(BJ) & {$[22-24]$} \\
B97-D3(BJ) & GGA & D3(BJ) & {$[22,25]$} \\
\hline SCAN-D3(BJ) & mGGA & D3(BJ) & {$[26,27]$} \\
M06L-D3 & mGGA & D3(0) & {$[28,29]$} \\
B97M-V & mGGA & VV10 & {$[30]$} \\
\hline B3LYP-D3(BJ) & single hyb. & D3(BJ) & {$[22,31,32]$} \\
$\omega B 97 X-V$ & single hyb. & VV10 & {$[33]$} \\
M052X-D3 & single hyb. & D3(0) & {$[29,34]$} \\
dlDF+D10 & single hyb. & DAS2010 & {$[35,36]$} \\
\hline DSD-BLYP-D3(BJ) & double hyb. & D3(BJ) & {$[37]$} \\
B2PLYP-D3(BJ) & double hyb. & D3(BJ) & {$[29,38]$} \\
PWPB95-NL & double hyb. & VV10 & {$[39-41]$} \\
PBE0DH-D3(BJ) & double hyb. & D3(BJ) & {$[42,43]$} \\
\hline
\end{tabular}

ranted inclusion. The three meta-GGAs were selected to investigate different dispersion correction forms, as well as the differences in approaches to their construction: a "non-empirical" representative in SCAN-D3(BJ), a combinatorially optimized B97M-V, as well as an "empirical" representative in M06L-D3. My selection of the four single hybrids followed similar patterns, with dlDF+D10 included as a counterpoint to M052X-D3: both DFAs are empirically fitted to data, but while in M052X shortand medium-range dispersion contributions are included, in dlDF they are excluded by design. Finally, the four double hybrids that I selected also range from DFAs with few optimized parameters (PBE0DH-D3(BJ)) to methods obtained from extensive fitting to data (DSD-BLYP-D3(BJ)).

To obtain HF and DFT energies of species at the basis set limit, I have carried out numerical finite element calculations in HelFEM. ${ }^{44]}$ As neither range-separated hybrids nor double hybrids are implemented in HelFEM, the selection of DFAs is limited to BLYP, PBE, revPBE, B97-D, SCAN, M06L, B97M-V, B3LYP, M052X, and dlDF. Note that the non-local "VV10" component of B97M$\mathrm{V}$ is also not yet implemented in HelFEM. The parameters that I used to obtain total energies with the diatomic code are determined for each molecule by the diatomic_cbasis tool, requesting $10^{-10}$ accuracy. A 150-point quadrature rule is used with each DFA.

To investigate the performance of the proposed extrapolation schemes, I chose the GMTKN55 bench- 
mark containing 1505 relative energies or 2462 single point calculations. ${ }^{[19]}$ Due to the large size of GMTKN55, I used its "diet" subset containing 100 relative energies or 240 single point calculations (denoted diet100). ${ }^{[45]}$ The latter has been shown to reproduce the results of the former both qualitatively and quantitatively, but its use is nonetheless re-validated below. Unlike in the original GMTKN55 study, ${ }^{[19]}$ here I used the same basis sets for all calculations, without augmentation in selected database subsets. All calculations are performed with Psi4 using development versions of $1.4,{ }^{[6]}$ with the default convergence criteria for energy and a $(75,302)$ point integration grid. Double hybrid functionals are applied without the frozen core approximation. The figure of merit for the GMTKN55 and diet100 benchmarks is the weighted mean absolute deviation (WTMAD), which corresponds to the average of the weighted absolute devations of computed relative energies from the reference energies in the dataset, with weights taken from the original references. ${ }^{[19,45]}$

To check performance of the extrapolation methods for energies of van der Waals complexes, I have turned to the NCDT dataset ${ }^{[46]}$ in it's revised form. ${ }^{[47]}$ A modified version of Psi4's cbs () driver function is used to obtain the interaction energies in an automated fashion.

All raw input and output files, collated results in json format, and Jupyter notebooks used to analyse the results and prepare the figures and tables in this manuscript are included in the Supplemental information archive.

\section{Results}

As modern DFAs contain various components with presumably different scalings, this is a good point to outline the nomenclature I use below. The total energy of a density functional $\left(E^{\mathrm{DFA}}\right)$, such as B2PLYP-D3(BJ), contains a single-reference energy component obtained from the self-consistent cycle including the exchange-correlation functional $\left(E^{\mathrm{fctl}}\right)$, double-hybrid correlation contribution $\left(\Delta E^{\mathrm{dh}}\right)$, and dispersion correction $\left(E^{\text {disp }}\right)$ :

$$
E^{\mathrm{DFA}}=E^{\mathrm{fctl}}+\Delta E^{\mathrm{dh}}+E^{\mathrm{disp}}
$$


For the B2PLYP-D3(BJ) example above, $E^{\text {fctl }}$ is the self-consistent density functional energy, which contains the nuclear repulsion, one- and two-electron energies, and the exchange-correlation component of the DFA. This single-reference energy contribution contains all components that affect the KohnSham orbitals, including the non-local correlation $\left(E^{\mathrm{nl}}\right)$ term, if present. The $\Delta E^{\mathrm{dh}}$ is non-zero only for double-hybrid functionals and contains the scaled MP2-like correlation. The dispersion term for the functionals in this study, including B2PLYP-D3(BJ), depends only parametrically on the functional, i.e. it is not affected by basis set size, and therefore shouldn't be further extrapolated. For MP2, the components in the above equation are simply equivalent to the H-F energy, the MP2 correlation energy, and zero, respectively.

\subsection{Hartree-Fock extrapolation revisited}

First I focus on the global $\alpha=1.63$ value determined for the exponential extrapolation (Eq. (2)) by Halkier et al., which was fitted using a set of 9 closed-shell diatomics. ${ }^{[5]}$ While the H-F energy converges exponentially with basis set size, ${ }^{[48,49]}$ this is a good point to check whether a power (Eq. (1)) or an expsqrt function (Eq. (3)) performs better than the exponential function when used with smaller basis sets. For this purpose I used an updated database of H-F energies of 70 diatomic species, obtained from numerical basis set calculations, which has been recently published. ${ }^{44]}$ This dataset includes many charged species, open shell diatomics, as well as species with elements from the third row of the periodic table. Due to the difficulty in convergence of ${ }^{3} \mathrm{VO}^{-}$and ${ }^{1} \mathrm{CrMn}^{+}$, these two molecules are excluded in the current study, forming a dataset of 68 species.

Fig. 1 presents the $\alpha$ s obtained from fits of the logarithms of Eqs. (1-3) to cc-pv $X$ z-pp data $(X \in$ $[\mathrm{d}, \mathrm{t}, \mathrm{q}, 5])$. Species, for which the total energy doesn't converge smoothly, or for which the basis set contains an ECP, are excluded from this analysis. The average values are $\bar{\alpha}=1.35 \pm 0.24$ for exponential, $\bar{\alpha}=4.36 \pm 0.75$ for power, and $\bar{\alpha}=4.91 \pm 0.86$ for expsqrt functions, with $n=57$. The median values $(\tilde{\alpha})$ are close to the means. As the scatter of the individual $\alpha$ s for the three extrapolation functions is very similar, with relative $\sigma_{\alpha}$ of around $18 \%$, the following discussion is focused only on the exponential extrapolation function.

While the $\tilde{\alpha}$ (as well as $\bar{\alpha}$ ) obtained here is significantly lower than the $\alpha=1.63$ proposed by Halkier 


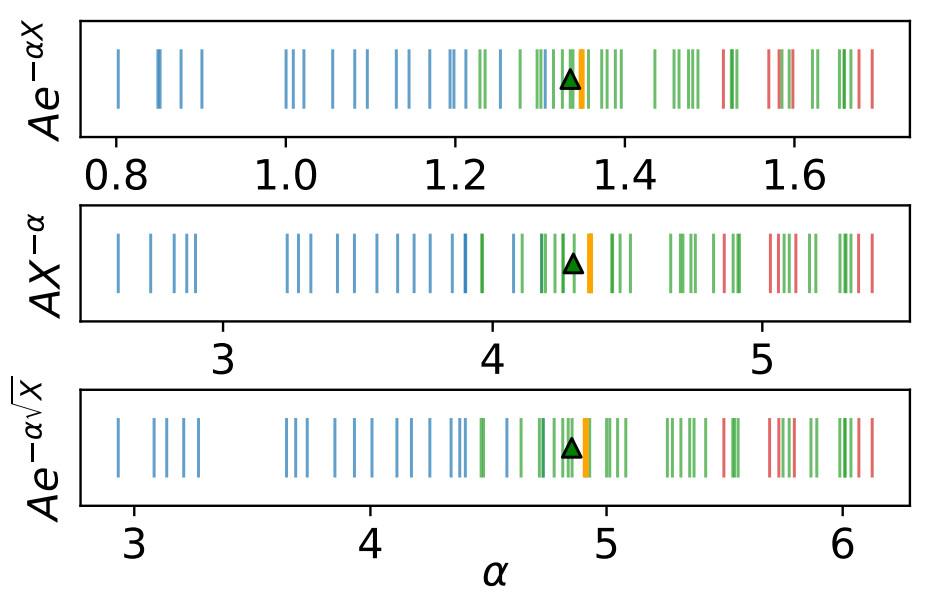

Figure 1: Values of $\alpha$ for various extrapolation functions obtained for the set of 68 diatomics, for the cc-pv $X$ z-pp basis set family. The means $(\bar{\alpha}, \mathbf{\Delta})$ and medians $(\tilde{\alpha}, \mid)$ also shown. Colours correspond to cationic (red), neutral (green), or anionic (blue) species.

et al., ${ }^{[5]}$ the distribution of charged species indicated by the colour-coding in Fig. 1 shows that anionic species converge to the basis set limit slower than neutral or cationic species. This has also been shown by Varandas, who highlighted that diffusely augmented basis sets increase the speed of convergence of anions, but that it is hard to predict which basis set will yield the best relative energies. ${ }^{[8]}$ The effect of different combinations of charges and multiplicities in this dataset is shown as a matrix in Fig. 2. There is no correlation of $\tilde{\alpha}$ with multiplicity, but there is a significant correlation with charge. For all three charge classes, the relative $\sigma_{\alpha}$ is significantly smaller than the $18 \%$ for the overall dataset. Therefore, at least for the cc-pv $X$ z-pp basis sets, it may be appropriate to use different $\alpha$ values for cationic (1.59), neutral (1.45), or anionic (1.08) species. As this charge-dependent effect is likely to be most pronounced in electron affinities and ionisation potentials, the G21EA and G21IP subsets of GMTKN55 can be used to validate this charge-dependent approach. In Table 2 I compare the $[2,3]-\zeta$ and $[3,4]-\zeta$ exponential extrapolations using a charge-independent $\tilde{\alpha}=1.35$ to an extrapolation using the charge-dependent values above. For the electron affinities, the WTMADs of the charge dependent extrapolation are lower than for the global approach; for the ionisation potentials the charge dependent approach is only beneficial at the $[3,4]-\zeta$ level.

The $\tilde{\alpha}$ values for exponential extrapolation with the four basis set families investigated in this study are presented in Table 3 . The pcseg- $N$ basis set family (where $X \approx N+1$ with $N \in[0-4]$ ) shows trends similar to the cc-pv $X z$ z-pp basis sets, but the magnitude of the variations in $\tilde{\alpha}$ results between the charge 
Table 2: Comparison of WTMAD values for databases of elecron affinities (G21EA) and ionisation potentials (G21IP) with exponential global and charge-dependent extrapolation of Hartee-Fock results using cc-pv $X z$-pp basis sets, with cc-pv5z-pp as reference.

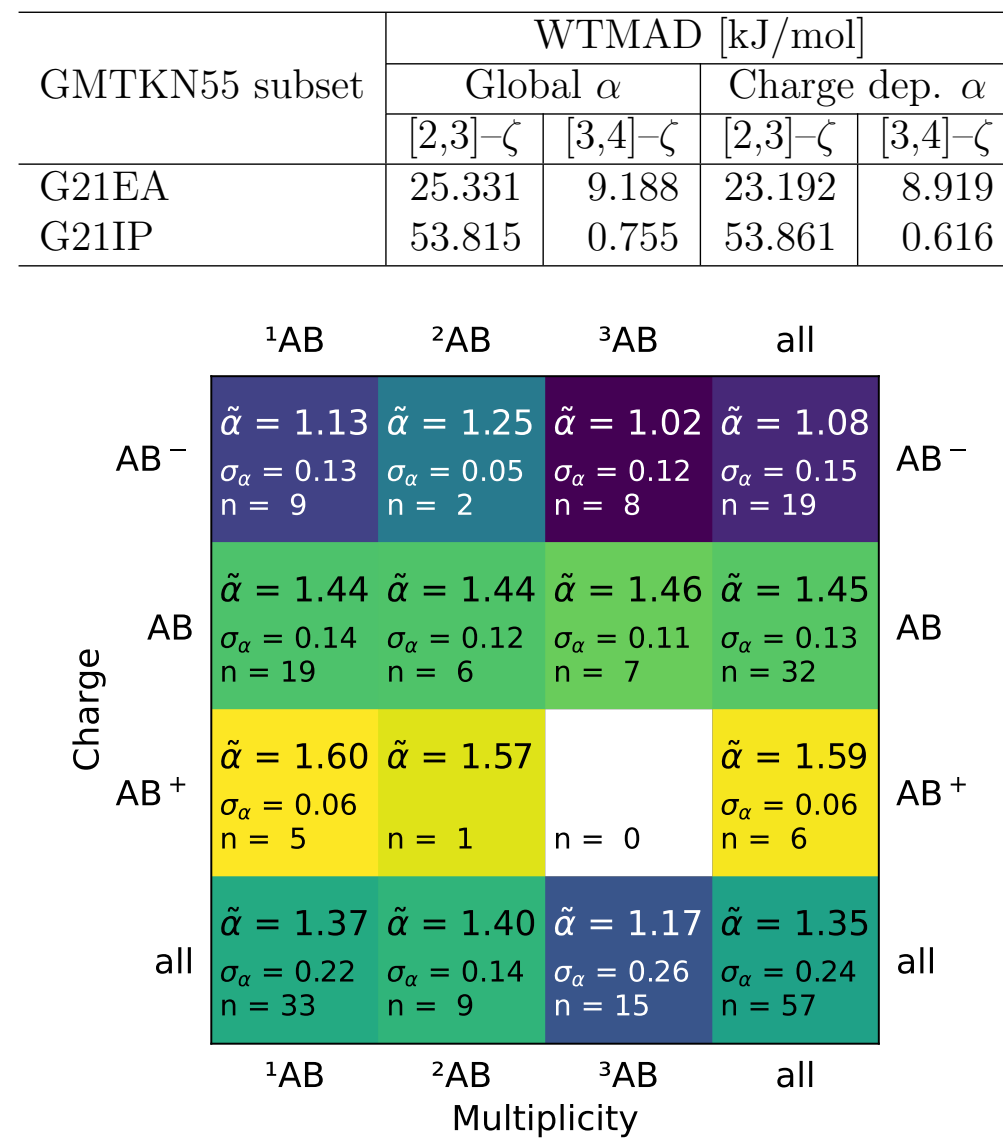

Figure 2: Matrix of values of $\tilde{\alpha}, \sigma_{\alpha}$, and $n$ for all combinations of charges and multiplicities in the dataset of diatomic molecules, for the cc-pv $X$ z-pp basis set family.

classes is significantly smaller. The overall $\tilde{\alpha}=1.26$ is comparable to the results from cc-pv $X$ z-pp basis sets. However, the distribution of the $\alpha$ values is almost bimodal as shown in Fig. 3, so the use of a single $\alpha$ may still be problematic. The Karlsruhe families converge much faster, with $\tilde{\alpha}$ being almost double that of pcseg- $N$ and cc-pv $X z$ z-pp. This could also be a consequence of fitting to [2,3,4]- $\zeta$ data. The inclusion of diffuse functions (def2-Xzvp $\rightarrow$ def2- $X$ zvpd) does not eliminate the scatter in the results for anions, but merely shifts the distribution to a higher mean. The extrapolations with power and expsqrt functions behave similarly.

Another approach to H-F extrapolation was proposed recently by Varandas. ${ }^{[48]}$ In summary, the method amounts to anchoring an exponential function (Eq. (2)), applicable for all basis set families and chemical systems with a universal $\alpha=2.284$, by adjusting $A$ to ensure that the fitting function passes 
Table 3: Comparison of $\tilde{\alpha} \pm \sigma_{\alpha}$ values for charge classes and basis set families using exponential extrapolation.

\begin{tabular}{l|r|r|r|r}
\hline \multirow{2}{*}{ Basis set family } & \multicolumn{4}{|c}{ Charge group } \\
\cline { 2 - 5 } & AB $^{+}$ & AB & AB $^{-}$ & All \\
\hline cc-pv $X$ z-pp & $1.59+/-0.06$ & $1.45+/-0.13$ & $1.08+/-0.15$ & $1.35+/-0.24$ \\
pcseg- $N$ & $1.57+/-0.08$ & $1.26+/-0.19$ & $1.21+/-0.19$ & $1.26+/-0.19$ \\
def2- $X$ vp & $2.58+/-0.11$ & $2.65+/-0.32$ & $2.11+/-0.38$ & $2.58+/-0.41$ \\
def2- $X$ vpd & $2.58+/-0.11$ & $2.71+/-0.12$ & $2.59+/-0.61$ & $2.70+/-0.37$ \\
\hline
\end{tabular}

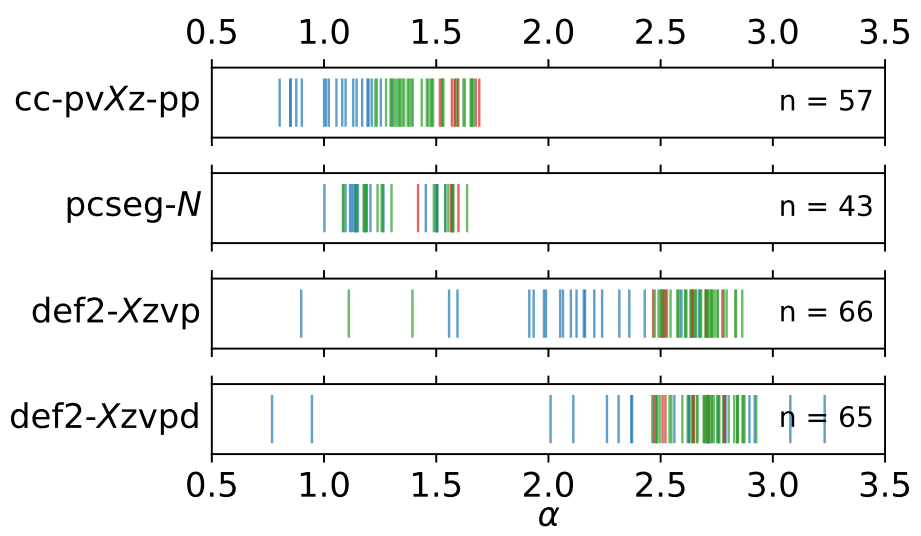

Figure 3: Values of $\alpha$ for the set of 68 diatomics shown for all studied basis set families. Colours correspond to cationic (red), neutral (green), or anionic (blue) species.

through the Hartree-Fock complete basis limit as well as the energy obtained with a STO-2G basis. The basis set cardinalities $(X)$ are then renormalised to hierarchical numbers $(\tilde{x})$, with $\tilde{x}=1$ for a STO-2G basis set. For the cc-pv $X z$ basis sets, the $\langle\tilde{x}\rangle$ values obtained from averaging over a set of 18 neutral, closed shell species are $3.02,3.64,4.28$, and 5.14 for $X \in[\mathrm{d}, \mathrm{t}, \mathrm{q}, 5]$, respectively. ${ }^{[4]}$ Unfortunately, neither Jensen nor Karlsruhe basis sets were investigated, and the regression was performed with $[6,7]-\zeta$ extrapolated data as reference values instead of numerical basis set limits. Here, I have re-fitted the hierarchical numbers $\tilde{x}$ using the dataset of the 68 diatomic species, by first calculating $A$ for each diatomic using the STO-2G result, and then calculating $\tilde{x}$ for each larger basis set, and finally averaging and obtaining $\langle\tilde{x}\rangle$ over the whole dataset. The $\langle\tilde{x}\rangle_{\mathrm{s}}$ are $3.59 \pm 0.54,4.08 \pm 0.53,4.58 \pm 0.58$, and $5.26 \pm 0.64$ for the cc-pv $X$ z-pp basis sets, which is significantly higher and with a significantly wider $\sigma_{\tilde{x}}$ than the results of Varandas. This is likely due to the inclusion of anionic species in the fitting process.

The performance of the discussed H-F extrapolation methods is presented in Table 4, using cc$\mathrm{pv}[\mathrm{d}, \mathrm{t}] \mathrm{z}-\mathrm{pp}$ data. In the diatomics dataset, the extrapolated values are compared to and normalised by the reference $E_{\infty}^{\mathrm{H}-\mathrm{F}}$ values obtained from Lehtola, ${ }^{[44]}$ with each species in the dataset weighted equally, 
Table 4: Performance of various H-F extrapolation methods in the diatomics dataset and the diet100 subset of GMTKN55, using cc-pv[d,t]z-pp data.

\begin{tabular}{l|l|l|l|r|r}
\hline \multicolumn{3}{c|}{ Extrapolation method } & Diatomics MRE & diet100 WTMAD \\
\hline Function & $\alpha$ & {$\left[X_{\mathrm{d}}, X_{\mathrm{t}}\right]$} & Ref. & $\%$ & $\mathrm{~kJ} / \mathrm{mol}$ \\
\hline exponential & 1.630 & {$[2.00,3.00]$} & {$[5]$} & 0.0061 & 12.21 \\
exponential & 1.450 & {$[2.00,3.00]$} & {$[5]$} & 0.0060 & 12.07 \\
power & 3.400 & {$[2.00,3.00]$} & {$[4]$} & 0.0016 & 5.76 \\
exponential & 2.284 & {$[3.02,3.64]$} & {$[48]$} & 0.0062 & 12.41 \\
exponential & 1.349 & {$[2.00,3.00]$} & c.w. & 0.0060 & 11.95 \\
power & 4.361 & {$[2.00,3.00]$} & c.w. & 0.0034 & 7.29 \\
expsquare & 4.912 & {$[2.00,3.00]$} & c.w. & 0.0026 & 6.18 \\
exponential & 2.284 & {$[3.59,4.08]$} & c.w. & 0.0059 & 12.07 \\
\hline
\end{tabular}

obtaining a mean relative error (MRE). For the diet100 subset of the GMTKN55 database, the respective cc-pv5z-pp values are taken as the reference for each of the 100 reactions, as the goal of this benchmark is to estimate the performance of the extrapolation method, not H-F theory as such. The best performing scheme overall is Truhlar's power extrapolation with $\alpha=3.4$ (see Eq. (1)), which was developed to match estimates of the H-F limit for Ne, HF, and $\mathrm{H}_{2} \mathrm{O}$ from $[2,3]-\zeta$ basis data. ${ }^{[4]}$ The exponential function performs significantly worse, whether a global $\alpha=1.63$ or $[2,3]-\zeta$ specific $\alpha=1.45$ is applied. ${ }^{[5]}$ Fitting extrapolation constants using a larger dataset, or using the approach of Varandas, ${ }^{[48]}$ fails to improve the performance in $[2,3]-\zeta$ extrapolation. From the four global fits to the 68 diatomics performed in this study, the best function for extrapolating cc-pv[d,t]z-pp data is the expsqrt function (see Eq. (3)).

\subsection{Extrapolation of functional energy in DFAs}

The H-F results show it is not necessary to use the large dataset of 68 diatomics, and that a wellperforming extrapolation formula can be obtained from fitting to basis set limit estimates of as little as 3 species, provided it is tailored for a $[2,3]-\zeta$ extrapolation. To determine the universality of $\alpha$ values in DFT, I have calculated the numerical basis set limit energies for the smaller set of 9 diatomics used by Halkier et al. ${ }^{[5]}$ The basis set limits are then used to obtain $\tilde{\alpha}$ for each functional using the cc-pv $X z$ z-pp basis sets, analogously to the above work for H-F. In addition to a "global" extrapolation using all available basis sets within the family, I have also included a "[d,t]" extrapolation using only results from $[2,3]-\zeta$ basis sets. Only the functional $\left(E^{\mathrm{fctl}}\right)$ component of the energy is extrapolated. For the B97M-V

functional, where $E^{\mathrm{fctl}}$ contains the non-local correlation $\left(E^{\mathrm{nl}}\right)$ term, this term is subtracted from the 

$E^{\text {fctl }}$ value to be directly comparable to the HelFEM results.

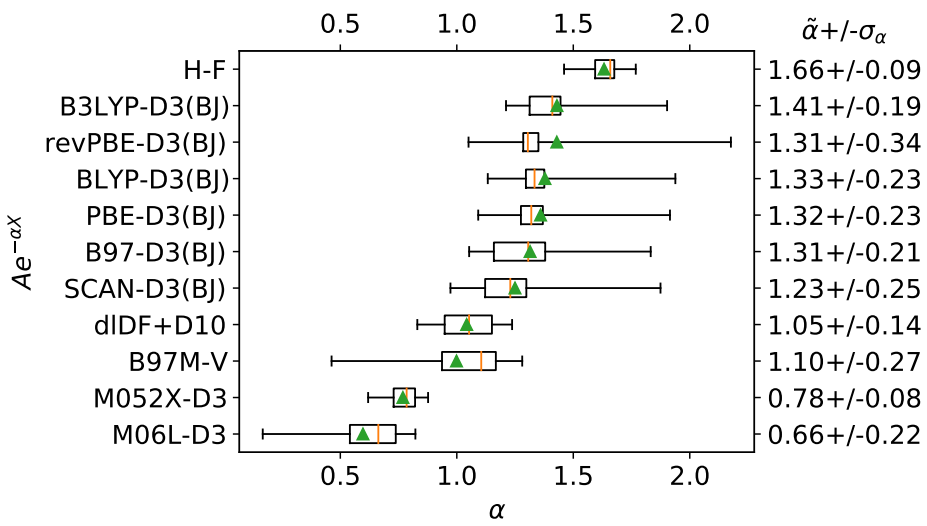

Figure 4: Box plots of $\alpha$ of the studied DFAs with cc-pv $X \mathrm{z}-\mathrm{pp}$ basis sets, for the reference dataset of 9 closed-shell diatomics of Halkier et al. The symbols are means $(\boldsymbol{\Delta})$ and medians $(\mid)$. The medians of each DFA are also listed on the right y-axis.

Box plots of the $\alpha$ s for each studied DFA are shown in Fig. 4, using an exponential extrapolation with the cc-pv $X z$ z-pp family of basis sets. The H-F result of $\bar{\alpha}=1.61(\tilde{\alpha}=1.66)$ agrees well with the reported data of Halkier et al. obtained with the same dataset, ${ }^{[5]}$ validating the current methodology. The two Minnesota DFAs are clear outliers, with $\tilde{\alpha}$ below 1.0. This can be partially attributed to the non-negligible amount of dispersion trained into both M06L and M052X, especially as the dispersionless dlDF, which uses a functional form similar to M052X, is much closer to the overall mean. The overall averages $(\langle\tilde{\alpha}\rangle)$ over all but Minnesota DFAs are $1.26 \pm 0.11$ for exponential, $4.06 \pm 0.34$ for power, and $4.58 \pm 0.40$ for expsqrt extrapolation functions in a "global" extrapolation; for a "[d,t]" extrapolation these values decrease to $1.26 \pm 0.07,3.12 \pm 0.17$, and $3.97 \pm 0.22$, respectively. Results for other basis set families are in the Supplemental material.

The effect of increasing amount of (Hatree-)Fock exchange (Fx) in the DFA on $\alpha$ is shown in Fig. 5, for a series of PBE-related functionals. With the exception of H-F which contains no correlation, only the percentages of Fock and PBE exchanges are modified, and the PBE correlation is always fully included. There is a clear trend of increasing $\bar{\alpha}$ and $\tilde{\alpha}$ with increasing proportion of Fock exchange. The $\sigma_{\alpha}$ decreases significantly with a higher percentage of Fx. A qualitatively similar trend can be seen between the BLYP and B3LYP data, and M06L and M052X data in Figure 4. The four GGA functionals investigated in this study (PBE, revPBE, BLYP, and B97-D) have a very similar $\bar{\alpha} \approx 1.28$, and the increase in $\bar{\alpha}$ between B3LYP (20\% Fx) and BLYP, $\Delta \bar{\alpha}=0.08$, is comparable to the difference between 
PBE0 (25\% Fx) and PBE for which $\Delta \bar{\alpha}=0.09$. From this data, I propose an exchange-dependent formula to obtain $\alpha$ for extrapolating the functional energy with Eq. (2): $\alpha=\alpha_{0}+\Delta \alpha \times f(\mathrm{Fx})$, where $f(\mathrm{Fx})$ is a linear function. Two sigmoidal functions (error and logistic) were also tested, but they do not improve the performance. Similar formulas can be derived for the power and expsqrt functions, and for the "[d,t]" extrapolation. For the Dunning and Karlsruhe basis set families, $\Delta \alpha$ is positive, while for the $\operatorname{pcseg}-N$ family, inclusion of Fx leads to a reduction in $\alpha$. See the Supplemental material for a complete overview.

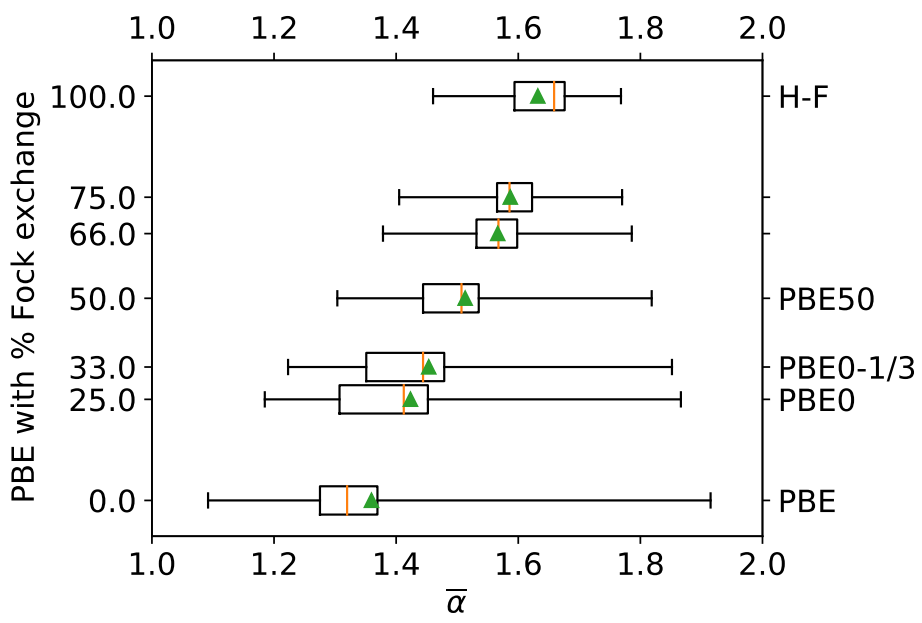

Figure 5: The relationship between $\bar{\alpha}$ and $\%$ of Fock exchange as part of total exchange in PBE. Note that $100 \%$ Fock exchange here corresponds to pure H-F. The means $(\boldsymbol{\Lambda})$ and medians $(\mid)$ are also shown. Calculated with the cc-pv $X$ z-pp basis set family.

\subsection{Extrapolation of the correlation energy in double hybrid DFAs}

As previously mentioned in the introduction, the convergence behaviour of the correlation energy in WFT is a much more studied phenomenon than the convergence of the functional energy, with Eq. (1) being the most widely used formula. The available literature on MP2 extrapolation is too large to review here; for a recent thorough investigation of MP2 convergence with various basis sets, I refer the reader to the recent work of Kirschner et al. ${ }^{[50]}$

As double hybrid DFAs contain an MP2 component, one would expect their convergence behaviour to be similar to that of MP2. However, in DFAs the correlation energy is evaluated using the KohnSham orbitals as opposed to the Hartree-Fock orbitals in MP2, so directly applying the same $\alpha$ may not be ideal. This raises the issue of obtaining reasonable estimates of basis set limits of the double-hybrid 
correlation $\left(\Delta E_{\infty}^{\mathrm{dh}}\right)$. Numerical estimates of the MP2 limit $\left(\Delta E_{\infty}^{\mathrm{MP} 2}\right)$ are not as common as for the H-F limit. ${ }^{[4]}$ Fortunately, Ranasinghe et al. ${ }^{[18]}$ have collated accurate literature values of the MP2 core-core and core-valence contributions from finite element calculations of atoms, ${ }^{[51-54]}$ and explicitly correlated calculations of molecules, ${ }^{[55]}$ and proposed a simple linear extrapolation method to obtain near-reference results from $[3,4,5]-\zeta$ calculations (Eq. (5)).

$$
\Delta E_{\infty}^{\mathrm{MP} 2} \approx \Delta E_{5 \mathrm{ZaPa}-\mathrm{CV}}^{\mathrm{MP2}}+\lambda \times\left(\Delta E_{4 \mathrm{ZaPa}-\mathrm{CV}}^{\mathrm{MP} 2}-\Delta E_{3 \mathrm{ZaPa}-\mathrm{CV}}^{\mathrm{MP} 2}\right), \quad \lambda=0.4307
$$

To prepare a set of $\Delta E_{\infty}^{\mathrm{dh}}$ data for each of the four functionals studied, I have collated the total MP2 correlation energies from the same dataset $\left(\mathrm{C}, \mathrm{N}, \mathrm{O}, \mathrm{F}, \mathrm{Ne}, \mathrm{Ar}, \mathrm{F}^{-}, \mathrm{Mg}, \mathrm{Be}, \mathrm{CH}_{2}, \mathrm{HF}, \mathrm{NH}_{3}, \mathrm{~F}_{2}, \mathrm{~N}_{2}\right.$ and $\mathrm{H}_{2} \mathrm{O}$ ). The valence-only MP2 correlation is then compared to the extrapolated values using frozen core calculations and the above extrapolation approach. ${ }^{[18]}$ The root mean square deviation (RMSD) of the two sets is $17.7 \mathrm{mEh}$, which is higher than the RMSD for core-core + core-valence correlation $(0.5 \mathrm{mEh})$, but sufficiently accurate for extrapolation purposes. For comparison, a two point cubic power extrapolation from [4,5]ZaPa-CV data has an RMSD of $18.1 \mathrm{mEh}$. Therefore, the estimated $\Delta E_{\infty}^{\text {dh }}$ values were calculated using Eq. (5) (see Supplemental information for the raw data).

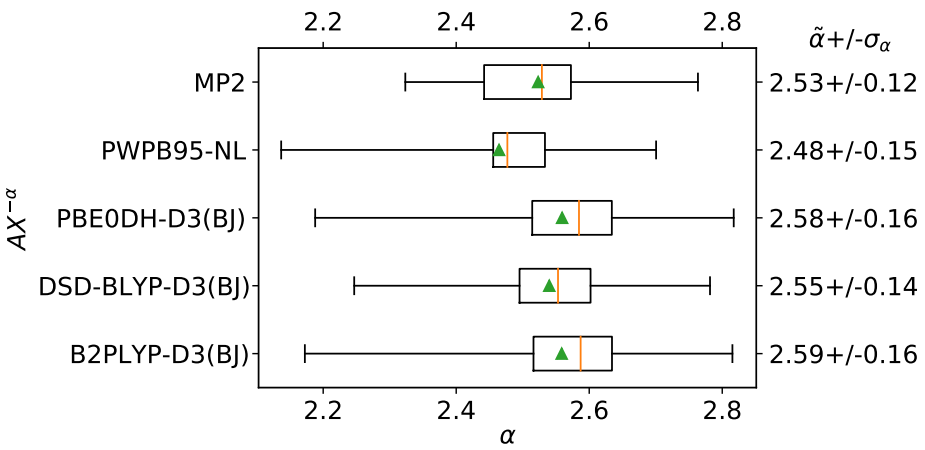

Figure 6: Box plots of $\alpha$ for the studied double-hybrid DFAs and MP2 with cc-pvXz-pp basis sets, for the reference dataset of atoms and small molecules of Ranasinghe et al. The symbols are means $(\boldsymbol{\Delta})$ and medians (|). The medians of each DFA are also listed on the right y-axis.

The four double-hybrid DFAs and MP2 have a very similar $\tilde{\alpha}$, as shown in Fig. 6. The convergence of MP2, with a global $\bar{\alpha}^{\mathrm{MP} 2}=2.53$ is significantly slower than the ideal cubic scaling. For the Dunning and Karlsruhe basis set families, the "[d,t]" fits of $\alpha^{\mathrm{dh}}$ are $\sim 0.3$ lower than the global fits; the Jensen 
basis sets are again an outlier for which the global fit is smaller. The "[d,t]" $\alpha^{\mathrm{MP} 2}$ values for Ne, HF, $\mathrm{H}_{2} \mathrm{O}$, and $\mathrm{N}_{2}$ are $2.18,2.21,2.37$, and 2.28 , which is in a good agreement with Truhlar's $\bar{\alpha}^{\mathrm{MP} 2}=2.2$ for the same species and basis sets, ${ }^{[4]}$ validating the current method.

\subsection{Performance of $[2,3]-\zeta$ extrapolation in GMTKN55}

To significantly reduce the number of calculations required for the benchmarking below, I resort to the use of the diet100 subset ${ }^{[45]}$ of the GMTKN55 dataset. ${ }^{[19]}$ The diet100 subset has been validated for all of the functionals studied by Goerigk et al., ${ }^{[19]}$ yielding good quantitative agreement between the diet100 and full GMTKN55 WTMADs. Here, I re-validate the use of diet100 as a proxy for GMTKN55 by considering the PBE-D3(BJ) functional and the def2-Xzvpd basis set family. The results presented in Table 5 show the discrepancy between the two benchmarks is small, with the largest deviation of $3 \mathrm{~kJ} / \mathrm{mol}$ occuring in the def2-qzvpd data. An agreement of the two sets to within $0.2 \mathrm{~kJ} / \mathrm{mol}$ in the extrapolated data validates the use of diet100 subset for this purpose.

Table 5: Comparison of WTMAD values of the diet100 subset and the full GMTKN55 database for the PBE-D3(BJ) functional, def2- $X$ zvpd basis sets, and selected extrapolation methods.

\begin{tabular}{l|r|r}
\hline \multirow{2}{*}{ Basis } & diet100 & GMTKN55 \\
\cline { 2 - 3 } & \multicolumn{2}{|c}{ WTMAD [kJ/mol] } \\
\hline def2-svpd & 64.3 & 62.8 \\
def2-tzvpd & 43.5 & 43.6 \\
def2-qzvpd & 42.6 & 45.6 \\
[s, t]zvpd ORCA $^{[8]}$ & 43.1 & 43.2 \\
[s, t]zvpd expsqrt $^{2}$ & 42.5 & 42.7 \\
\hline
\end{tabular}

As comparing the extrapolation results of all methods with all studied functionals for all four basis set families would be tedious, an overview of the performance of 13 extrapolation methods with the cc-pv $X$ z-pp basis sets and a smaller set of functionals is shown in Table 6. Results for other basis set families are included in Supplemental material. The extrapolated results are listed along nonextrapolated values calculated with $3-$ and $4-\zeta$ basis sets. A well performing $[2,3]-\zeta$ extrapolation should approach $4-\zeta$ results. The exponential function performs rather poorly in DFT extrapolations, with the extrapolated BLYP-D3(BJ) and B3LYP-D3(BJ) results barely beating 3- $\zeta$ data, while for the double-hybrid B2PLYP-D3(BJ) it performs even worse. Scaling $\alpha$ based on Fx also shows no benefit, 
whether $f(\mathrm{Fx})$ is a linear or sigmoidal function, and will not be considered further. Unsurprisingly, the $[\mathrm{d}, \mathrm{t}]$ fits perform better than global fits. Truhlar's extrapolation formula for MP2 performs surprisingly well for DFT, outperforming the $[\mathrm{d}, \mathrm{t}]$ defaults used in ORCA, ${ }^{[8]}$ obtaining values within $1.5 \mathrm{~kJ} / \mathrm{mol}$ of $4-\zeta$ results, despite the small training set $\left(\mathrm{Ne}, \mathrm{HF}, \mathrm{H}_{2} \mathrm{O}\right)$ used in its development. The $[\mathrm{d}, \mathrm{t}]$ variants of the power and expsqrt functions are the best perfomers, with results within $0.3 \mathrm{~kJ} / \mathrm{mol}$ of the $4-\zeta$ results in BLYP and B3LYP. The power formula is more convenient, as it uses the same extrapolation function for both $E^{\mathrm{fctl}}$ and $\Delta E^{\mathrm{dh}}$ with different $\alpha$ s. Similar trends are present for the other three basis set families. I should note the improvement between cc-pvtz-pp and cc-pvqz-pp is on the order of $10 \mathrm{~kJ} / \mathrm{mol}$ in WTMAD, while for the Karlsruhe and Jensen basis sets it is well below $5 \mathrm{~kJ} / \mathrm{mol}$. For these families, the global expsqrt fit performs slightly better than all other methods, with the extrapolated WTMAD generally halfway between $3-$ and $4-\zeta$ results.

Table 6: Comparison of WTMAD values in the diet100 subset of the GMTKN55 database for the proposed extrapolation methods for representatives of three classes of DFAs: a GGA (BLYP-D3(BJ)), single-hybrid (B3LYP-D3(BJ)), and double-hybrid (B2PLYP-D3(BJ)).

\begin{tabular}{l|l|l|l|r|r|r}
\hline \multirow{2}{*}{ Basis } & \multirow{2}{*}{ Function } & \multirow{2}{*}{$\alpha$} & \multicolumn{2}{|c}{ diet100 WTMAD [kJ/mol] } \\
\cline { 5 - 7 } & & & - & BLYP-D3(BJ) & B3LYP-D3(BJ) & B2PLYP-D3(BJ) \\
\hline cc-pvtz-pp & - & - & $2.200^{[4]}$ & 46.0 & 41.3 & 28.7 \\
cc-pv[d,t]z-pp & power [d,t] & $3.400^{[4]}$ & $2.400^{[8]}$ & 47.0 & 33.4 & 23.7 \\
cc-pv[d,t]z-pp & expsqrt [d,t] & $4.420^{[8]}$ & 2.550 & 55.3 & 33.7 & 23.7 \\
cc-pv[d,t]z-pp & exponential global & 1.258 & 2.257 & 55.3 & 40.7 & 30.0 \\
cc-pv[d,t]z-pp & exponential [d,t] & 1.263 & 2.550 & 49.4 & 35.6 & 30.4 \\
cc-pv[d,t]z-pp & power global & 4.062 & 2.257 & 45.7 & 32.3 & 25.1 \\
cc-pv[d,t]z-pp & power [d,t] & 3.115 & 2.550 & 47.8 & 34.1 & 22.5 \\
cc-pv[d,t]z-pp & expsqrt global & 4.577 & 2.257 & 45.7 & 32.3 & 23.7 \\
cc-pv[d,t]z-pp & expsqrt [d,t] & 3.974 & 55.5 & 40.9 & 22.5 \\
cc-pv[d,t]z-pp & exponential global & $1.322+0.373 \times f(\mathrm{Fx})$ & 2.550 & 55.4 & 40.8 & 30.3 \\
cc-pv[d,t]z-pp & exponential [d,t] & $1.296+0.095 \times f(\mathrm{Fx})$ & 2.257 & 36.6 & 30.5 \\
cc-pv[d,t]z-pp & power global & $4.254+1.160 \times f(\mathrm{Fx})$ & 2.550 & 50.0 & 32.8 & 26.8 \\
cc-pv[d,t]z-pp & power [d,t] & $3.195+0.233 \times f(\mathrm{Fx})$ & 2.257 & 46.1 & 35.3 & 23.2 \\
cc-pv[d,t]z-pp & expsqrt global & $4.802+1.334 \times f(\mathrm{Fx})$ & 2.550 & 48.4 & 25.7 \\
cc-pv[d,t]z-pp & expsqrt [d,t] & $4.076+0.298 \times f(\mathrm{Fx})$ & 2.257 & 46.1 & 32.8 & 23.2 \\
cc-pvqz-pp & - & - & - & 45.5 & 32.0 & 21.0 \\
\hline
\end{tabular}

The performance of selected functionals combined with the cc-pv $X \mathrm{z}-\mathrm{pp}$ basis sets in the diet100 subset of GMTKN55 is shown in Fig. 7. The figure contains results obtained using the power $[\mathrm{d}, \mathrm{t}]$ extrapolation (•) and global expsqrt extrapolation (o) in addition to all cc-pv $X z$-pp results. The dlDF+D10 functional is not included as its performance in the diet100 benchmark is very poor: the dispersion correction in this functional is only defined for multi-fragment species. In most cases, the $[\mathrm{d}, \mathrm{t}]$ extrapolation 


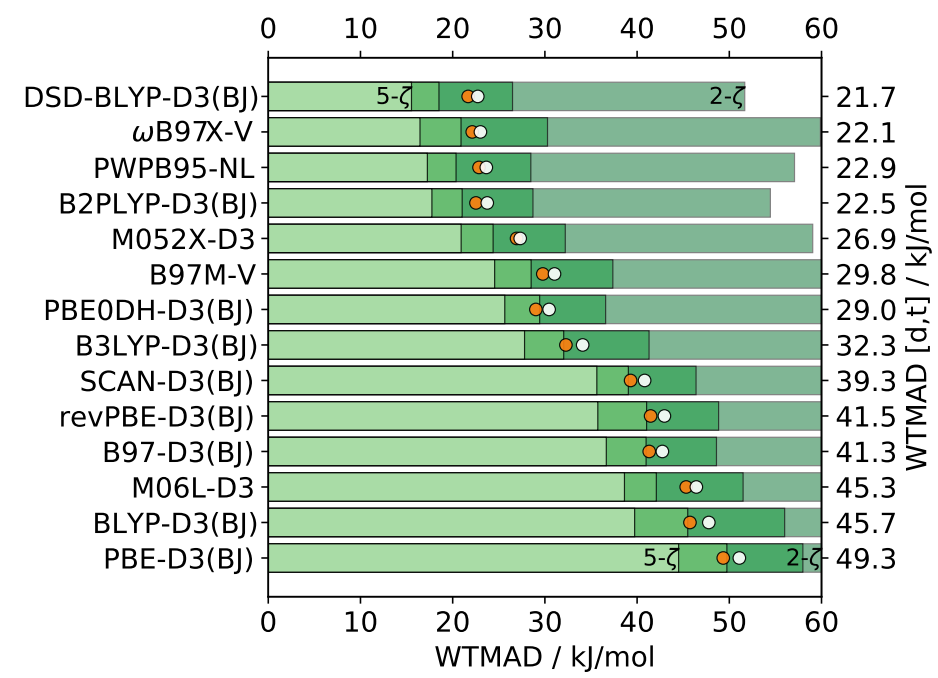

Figure 7: The WTMAD values for the diet100 dataset obtained with the listed DFAs using cc-pv $X \mathrm{z}-\mathrm{pp}$ basis sets $(X \in[\mathrm{d}, \mathrm{t}, \mathrm{q}, 5]$, green bars). The extrapolated cc-pv[d,t]z-pp results (• for $[\mathrm{d}, \mathrm{t}]$ power, o for global expqsrt) are also included, with the WTMAD shown on the right y-axis.

brings performance in line with cc-pvqz-pp results, the notable exceptions are the Minnesota functionals (M06L-D3 and M052X-D3) due to their different scaling of the functional energy (see Fig. 4), and some of the double hybrids. In all cases, the improvement achieved by cc-pv[dt]z-pp extrapolation over pure cc-pvtz-pp results is significant.
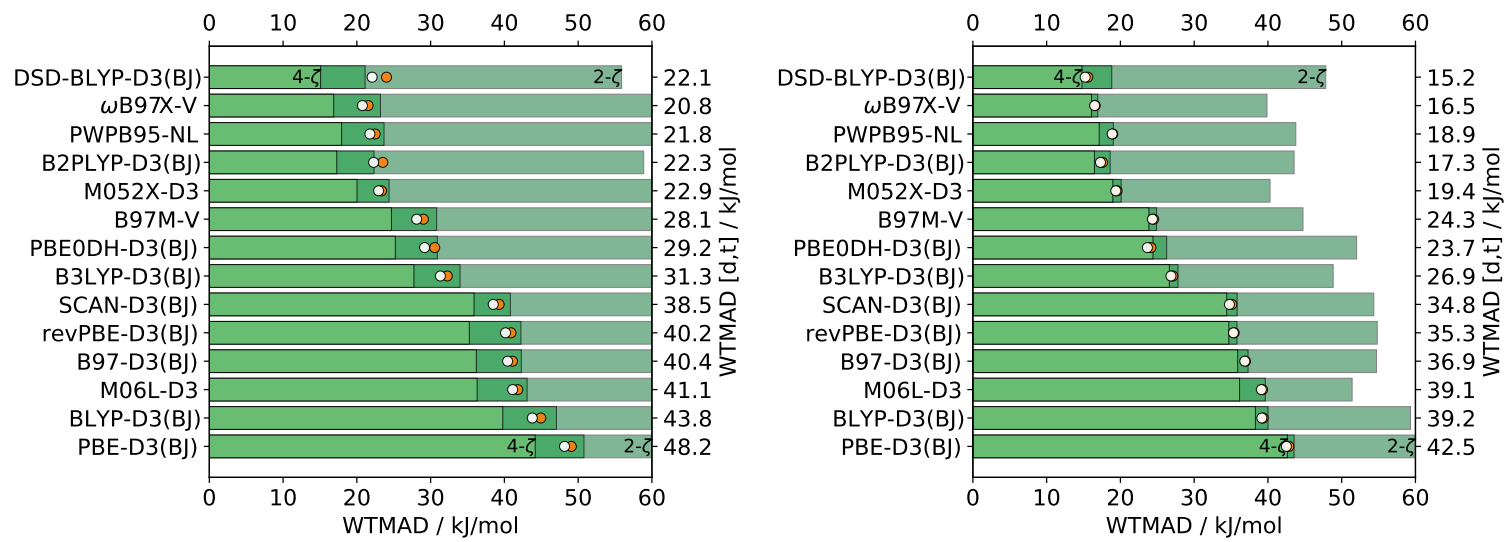

Figure 8: The WTMAD values for the diet100 dataset with def2- $X$ zvp (left) and def2- $X$ zvpd (right). Legend as in Fig. 7.

Analogous figures for the def2- $X$ zvp and def2- $X$ zvpd basis sets are shown in Fig. 8, the pcseg- $N$ results are included in the Supplemental information archive. In the def2-Xzvp and pcseg- $N$ series, neither of the extrapolation methods is particularly effective. Especially the DSD-BLYP-D3(BJ) and B2PLYPD3(BJ) results are troubling: the double hybrid performance can be slightly improved $(1.5 \mathrm{~kJ} / \mathrm{mol}$ 
reduction in WTMAD) by increasing $\alpha^{\mathrm{dh}}$ from 2.382 to 3.0 , but even then the extrapolation barely outperforms def2-tzvp results. Extrapolation of def2-[s,t]zvp or pcseg-[1,2] results therefore cannot be recommended. Upon diffuse augmentation, the difference between $3-\zeta$ and $4-\zeta$ results shrinks further. However, this time the global expsqrt extrapolation consistently improves upon def2-tzvpd results, with the exception of PWPB95-NL which remains near def2-tzvpd performance.

\subsection{Automated $[2,3]-\zeta$ extrapolation of non-covalent interaction energies}

While the GMTKN55 database contains several subsets of non-covalent interaction energies, I have decided to use an independent dataset to check the performance for non-covalent energies and also gather timing data in an automated fashion. For this purpose, I have modified the $\operatorname{cbs}()$ routine of Psi4 to accept DFAs, decompose them into $\mathrm{fctl}$, dh, and disp stages consistent with Eq. (4), and allow independent extrapolation of these components.

Table 7: Comparison of RMSD values in the NCDT dataset of non-covalent energies, with cc-pv $X \mathrm{z}$-pp basis sets. Extrapolation with $[\mathrm{d}, \mathrm{t}]$ power function, $\alpha=3.115, \alpha^{\mathrm{dh}}=2.257$. Timing is the ratio of total execution times of cc-pv[dt]z-pp versus cc-pvqz-pp.

\begin{tabular}{l|r|r|r|r}
\hline \multirow{2}{*}{ Functional } & \multicolumn{3}{|c|}{ NCDT RMSD [kJ/mol] } & \multirow{2}{*}{ Timing } \\
\cline { 2 - 4 } & cc-pvtz-pp & cc-pvqz-pp & cc-pv[dt]z-pp & \\
\hline BLYP-D3(BJ) & 7.01 & 4.84 & 4.81 & $83.8 \%$ \\
PBE-D3(BJ) & 8.86 & 6.73 & 6.78 & $81.3 \%$ \\
revPBE-D3(BJ) & 5.92 & 4.44 & 4.56 & $81.5 \%$ \\
B97-D3(BJ) & 5.61 & 4.14 & 4.17 & $83.2 \%$ \\
\hline SCAN-D3(BJ) & 9.06 & 7.53 & 7.62 & $67.0 \%$ \\
M06L-D3 & 4.29 & 3.18 & 3.54 & $66.1 \%$ \\
B97M-V & 5.36 & 3.48 & 4.07 & $138.7 \%$ \\
\hline B3LYP-D3(BJ) & 6.96 & 4.87 & 4.81 & $81.5 \%$ \\
M052X-D3 & 6.02 & 4.30 & 4.33 & $46.6 \%$ \\
dIDF+D10 & 8.28 & 9.42 & 9.28 & $66.1 \%$ \\
$\omega$ B97X-V & 5.03 & 2.84 & 3.21 & $152.4 \%$ \\
\hline B2PLYP-D3(BJ) & 5.13 & 2.96 & 3.60 & $81.9 \%$ \\
DSD-BLYP-D3(BJ) & 5.31 & 3.31 & 4.31 & $88.5 \%$ \\
PBE0DH-D3(BJ) & 7.20 & 5.60 & 5.84 & $94.5 \%$ \\
PWPB95-NL & 4.05 & 2.00 & 2.56 & $151.1 \%$ \\
\hline
\end{tabular}

Table 7 presents the results for cc-pv $X \mathrm{z}-\mathrm{pp}$ basis sets, with the cc-pv $[\mathrm{d}, \mathrm{t}] \mathrm{z}-\mathrm{pp}$ extrapolation performed using the $[\mathrm{d}, \mathrm{t}]$ power formula from Table 6 . For most GGAs, meta-GGAs, and single hybrids, the RMSD of the extrapolated results is almost identical to the RMSD of cc-pvqz-pp data at $\sim 80 \%$ of the 
computational cost. The exceptions are: i) extrapolated M06L-D3, which would benefit from a lower $\alpha$ (see Fig. 4); ii) dlDF+D10, where cc-pvtz-pp performs better than both cc-pvqz-pp and cc-pv[dt]z-pp; and iii) extrapolated B97M-V and $\omega \mathrm{B} 97 \mathrm{X}-\mathrm{V}$, which are $50 \%$ slower than cc-pvqz-pp due to the cost of evaluation of the VV10 contribution as part of the self-consistent cycle. This issue also affects PWPB95NL. However, the $E^{\mathrm{nl}}$ contribution can be evaluated as an additive correction at no loss to accuracy. ${ }^{[56]}$ Further speedups may be possible, if for example the converged wavefunction at the smaller basis set was used as an initial guess for the larger basis set. The improvement of the $[\mathrm{d}, \mathrm{t}]$ extrapolation over cc-pvtzpp results in double hybrids is generally worse than for the other DFAs, and can be only recommended for larger systems, where the cost of cc-pvqz-pp calculations would be prohibitively expensive: the largest system in the NCDT dataset is DMS-SO ${ }_{2}$, for which the computational time of B2PLYP-D3(BJ)/ccpv[dt]z-pp is $56 \%$ of B2PLYP-D3(BJ)/cc-pvqz-pp.

The performance of def2-[s,t]zvp extrapolation (global expsqrt fit) for the NCDT dataset is in line with the GMTKN55 results above: the RMSDs of def2-[s,t]zvp are not worse than for def2-tzvp, but def2qzvp performance is not achieved. In any case, the use of large basis sets without diffuse augmentation is questionable when accurate non-covalent interaction energies are required. ${ }^{[57]}$ For the same reason, I will omit the analysis of pcseg- $N$ with the NCDT dataset; the results for both basis set families are tabulated in the Supplemental material. Upon augmentation with diffuse functions, the performance of def2-[s,t]zvpd extrapolation (also global expsqrt fit) for non-covalent energies is a positive surprise: the RMSDs of def2-qzvpd are matched, if not outperformed, by the extrapolated results (see Table 8). $\mathrm{dlDF}+\mathrm{D} 10$ is again an outlier, as its performance decreases when going from $3-\zeta$ to a $4-\zeta$ basis set.

\subsection{Note on non-local correlation correction}

As the -NL (or -V) non-local corrections are included in the $E^{\mathrm{fctl}}$ component, they are extrapolated using the same formula and $\alpha$ as the rest of the functional energy. Results for B97M-V presented in Fig. 4 show a comparably large spread and a lower $\alpha$ than all other non-Minnesota functionals. The $E^{\mathrm{nl}}$ term seems to scale almost linearly in the cc-pv $X \mathrm{z}$-pp basis set family, regardless of the integration grid used. The DFAs including the VV10 term are some of the best performing functionals, but they do not seem to benefit from basis set extrapolation nearly as much as other functionals. The most obvious 
Table 8: Comparison of RMSD values in the NCDT dataset of non-covalent energies, with def2- $X$ zvpd basis sets. Extrapolation with a global expsqrt function, $\alpha=7.886, \alpha^{\mathrm{dh}}=2.267$. Timing is the ratio of total execution times of def2-[s,t]zvpd versus def2-qzvpd.

\begin{tabular}{l|r|r|r|r}
\hline \multirow{2}{*}{ Functional } & \multicolumn{3}{|c|}{ NCDT RMSD [kJ/mol] } & \multirow{2}{*}{ Timing } \\
\cline { 2 - 4 } & def2-tzvpd & def2-qzvpd & def2-[st]zvpd & \\
\hline BLYP-D3(BJ) & 4.09 & 4.04 & 3.98 & $75.8 \%$ \\
PBE-D3(BJ) & 5.15 & 5.08 & 4.89 & $78.0 \%$ \\
revPBE-D3(BJ) & 4.30 & 4.29 & 4.22 & $80.6 \%$ \\
B97-D3(BJ) & 3.91 & 3.90 & 3.82 & $77.8 \%$ \\
\hline SCAN-D3(BJ) & 6.57 & 6.45 & 6.32 & $62.4 \%$ \\
M06L-D3 & 2.86 & 2.95 & 2.75 & $61.4 \%$ \\
B97M-V & 2.71 & 2.68 & 2.52 & $132.5 \%$ \\
\hline B3LYP-D3(BJ) & 3.74 & 3.66 & 3.52 & $76.5 \%$ \\
M052X-D3 & 3.61 & 3.46 & 3.39 & $66.2 \%$ \\
dlDF+D10 & 9.88 & 10.16 & 10.08 & $65.6 \%$ \\
$\omega B 97 X-V$ & 1.37 & 1.16 & 1.20 & $150.7 \%$ \\
\hline B2PLYP-D3(BJ) & 1.82 & 1.85 & 1.53 & $77.1 \%$ \\
DSD-BLYP-D3(BJ) & 2.41 & 2.48 & 1.90 & $77.6 \%$ \\
PBE0DH-D3(BJ) & 4.65 & 4.66 & 4.30 & $77.5 \%$ \\
PWPB95-NL & 1.40 & 1.28 & 1.38 & $136.7 \%$ \\
\hline
\end{tabular}

case is in the NCDT results of PWPB95-NL and $\omega$ B97X-V with def2-Xzvpd basis sets. It may well be better to scale the $E^{\mathrm{nl}}$ term separately from $E^{\mathrm{fctl}}$, however, further research in this area is necessary.

\section{Conclusion}

There is no universal recipe for basis set extrapolation in density functional theory. In selected cases, such as for cc-pv $X z$-pp or def2- $X$ zvpd basis set families, a [2,3]- $\zeta$ extrapolation is a cost-efficient approach of obtaining results of nearly $4-\zeta$ quality, and I can recommend its use for cases where a $4-\zeta$ calculation is prohibitively expensive. However, the recipes are specific to each basis set family, with the cc-pv $X \mathrm{z}-\mathrm{pp}$ sets performing better with a power function fitted to $[\mathrm{d}, \mathrm{t}]$ data with $\alpha=3.115$, and the def2- $X$ zvpd sets with an exponential-square-root function with $\alpha=7.886$. I cannot recommend extrapolating pcseg- $N$ or def2-Xzvp data.

The MP2-like correction present in double hybrid density functionals can be extrapolated using a power function with $\alpha^{\mathrm{dh}}=2.257$ for cc-pv $X \mathrm{z}$-pp, and 2.267 for def2-Xzvpd. Despite the use of different orbitals, the $\Delta E^{\mathrm{dh}}$ scales comparably to $\Delta E^{\mathrm{MP} 2}$. A keen reader will notice the similarity of these results 
with Truhlar's proposed MP2 scaling of 2.2. ${ }^{[4]}$

The optimal extrapolation method for Hartree-Fock in $[2,3]-\zeta$ basis sets is not exponential, despite the exponential convergence of Hartree-Fock towards the complete basis set limit. The power function of Truhlar ${ }^{[4]}$ tailored for cc-pv[d,t]z extrapolations with an $\alpha=3.4$ remains the best performer given this set of requirements. The ideal global $\alpha$ for extrapolation of Hartree-Fock energies of diatomic molecules correlates with system charge, but is independent of system multiplicity.

The development of optimal extrapolation parameters for other basis set families can be considerably accelerated by fitting to the basis set limit data, calculated for several functionals in this work. I have shown that the dataset of 9 diatomics used by Halkier et al. ${ }^{[5]}$ is more than sufficient for extrapolation purposes. While there is a correlation between the amount of Fock exchange in the density functional approximation and the optimal extrapolation $\alpha$, scaling formulas that take the amount of Fock exchange into account do not perform as well as functional-independent $\alpha$ s. The modified cbs() routine in Psi4 allows for an intuitive and automated use of extrapolated density functional theory in calculations of energies, gradients, and Hessians.

\section{Acknowledgements}

I would like to thank Susi Lehtola for his help with HelFEM, Julian Gale for comments and feedback, and the Forrest Research Foundation for funding. This work was supported by resources provided by the Pawsey Supercomputing Centre (project f97) and the National Computational Infrastructure (project f97), with funding from the Australian Government and the Government of Western Australia.

\section{References}

[1] T. Helgaker, W. Klopper, H. Koch, and J. Noga, "Basis-set convergence of correlated calculations on water," The Journal of Chemical Physics, vol. 106, pp. 9639-9646, June 1997.

[2] D. Feller, "Application of systematic sequences of wave functions to the water dimer," The Journal of Chemical Physics, vol. 96, pp. 6104-6114, Apr. 1992. 
[3] C. E. Warden, D. G. A. Smith, L. A. Burns, U. Bozkaya, and C. D. Sherrill, "Efficient and automated computation of accurate molecular geometries using focal-point approximations to large-basis coupled-cluster theory," J. Chem. Phys., vol. 152, p. 124109, Mar. 2020.

[4] D. G. Truhlar, "Basis-set extrapolation," Chemical Physics Letters, vol. 294, pp. 45-48, Sept. 1998.

[5] A. Halkier, T. Helgaker, P. Jørgensen, W. Klopper, and J. Olsen, "Basis-set convergence of the energy in molecular Hartree-Fock calculations," Chemical Physics Letters, vol. 302, pp. 437-446, Mar. 1999.

[6] D. G. A. Smith, L. A. Burns, A. C. Simmonett, R. M. Parrish, M. C. Schieber, R. Galvelis, P. Kraus, H. Kruse, R. Di Remigio, A. Alenaizan, A. M. James, S. Lehtola, J. P. Misiewicz, M. Scheurer, R. A. Shaw, J. B. Schriber, Y. Xie, Z. L. Glick, D. A. Sirianni, J. S. O’Brien, J. M. Waldrop, A. Kumar, E. G. Hohenstein, B. P. Pritchard, B. R. Brooks, H. F. Schaefer, A. Y. Sokolov, K. Patkowski, A. E. DePrince, U. Bozkaya, R. A. King, F. A. Evangelista, J. M. Turney, T. D. Crawford, and C. D. Sherrill, "Psi4 1.4: Open-source software for high-throughput quantum chemistry," J. Chem. Phys., vol. 152, p. 184108, May 2020.

[7] J. F. Stanton, J. Gauss, L. Cheng, M. E. Harding, D. A. Matthews, and P. G. Szalay, "CFOUR, coupled-cluster techniques for computational chemistry, a quantum-chemical program package. Version 2.1," July 2019.

[8] F. Neese, "Software update: The ORCA program system, version 4.0," WIREs Computational Molecular Science, vol. 8, no. 1, p. e1327, 2018.

[9] A. J. Varandas, "Straightening the hierarchical staircase for basis set extrapolations: A low-cost approach to high-accuracy computational chemistry," Annu. Rev. Phys. Chem., vol. 69, pp. 177203, Apr. 2018.

[10] K. S. Raymond and R. A. Wheeler, "Compatibility of correlation-consistent basis sets with a hybrid Hartree-Fock / density functional method," Journal of Computational Chemistry, vol. 20, no. 2, pp. 207-216, 1999. 
[11] F. Jensen, "Polarization consistent basis sets. II. Estimating the Kohn-Sham basis set limit," The Journal of Chemical Physics, vol. 116, pp. 7372-7379, May 2002.

[12] B. C. Cabral and S. Canuto, "The enthalpy of the $\mathrm{O}-\mathrm{H}$ bond homolytic dissociation: Basis-set extrapolated density functional theory and coupled cluster calculations," Chemical Physics Letters, vol. 406, pp. 300-305, May 2005.

[13] N. Mardirossian and M. Head-Gordon, "Characterizing and understanding the remarkably slow basis set convergence of several Minnesota density functionals for intermolecular interaction energies," J. Chem. Theory Comput., vol. 9, pp. 4453-4461, Oct. 2013.

[14] Y.-Y. Chuang and S.-M. Chen, "Infinite basis set extrapolation for double hybrid density functional theory 1: Effect of applying various extrapolation functions," J. Comput. Chem., vol. 32, pp. 16711679, June 2011.

[15] Y.-Y. Chuang and S.-M. Chen, "Infinite basis set extrapolation for double hybrid density functional theory 2: Effect of adding diffuse basis functions," Jnl Chinese Chemical Soc, vol. 59, pp. 1094-1103, Sept. 2012.

[16] F. Jensen, "How large is the elephant in the density functional theory room?," J. Phys. Chem. A, vol. 121, pp. 6104-6107, Aug. 2017.

[17] D. Feller and D. A. Dixon, "Density functional theory and the basis set truncation problem with correlation consistent basis sets: Elephant in the room or mouse in the closet?," J. Phys. Chem. A, vol. 122, pp. 2598-2603, Mar. 2018.

[18] D. S. Ranasinghe, M. J. Frisch, and G. A. Petersson, "Core-core and core-valence correlation energy atomic and molecular benchmarks for Li through Ar," The Journal of Chemical Physics, vol. 143, p. 214110, Dec. 2015.

[19] L. Goerigk, A. Hansen, C. Bauer, S. Ehrlich, A. Najibi, and S. Grimme, "A look at the density functional theory zoo with the advanced GMTKN55 database for general main group thermochemistry, kinetics and noncovalent interactions," Phys. Chem. Chem. Phys., vol. 19, no. 48, pp. 32184-32215, 2017. 
[20] A. D. Becke, "Density-functional exchange-energy approximation with correct asymptotic behavior," Phys. Rev. A, vol. 38, pp. 3098-3100, Sept. 1988.

[21] C. Lee, W. Yang, and R. G. Parr, "Development of the Colle-Salvetti correlation-energy formula into a functional of the electron density," Phys. Rev. B, vol. 37, pp. 785-789, Jan. 1988.

[22] S. Grimme, S. Ehrlich, and L. Goerigk, "Effect of the damping function in dispersion corrected density functional theory," J. Comput. Chem., vol. 32, pp. 1456-1465, May 2011.

[23] J. P. Perdew, K. Burke, and M. Ernzerhof, "Generalized gradient approximation made simple," Phys. Rev. Lett., vol. 77, pp. 3865-3868, Oct. 1996.

[24] Y. Zhang and W. Yang, "Comment on "Generalized gradient approximation made simple"," Phys. Rev. Lett., vol. 80, pp. 890-890, Jan. 1998.

[25] S. Grimme, "Semiempirical GGA-type density functional constructed with a long-range dispersion correction," Journal of computational chemistry, vol. 27, no. 15, pp. 1787-1799, 2006.

[26] J. Sun, A. Ruzsinszky, and J. P. Perdew, "Strongly constrained and appropriately normed semilocal density functional," Phys. Rev. Lett., vol. 115, p. 036402, July 2015.

[27] J. G. Brandenburg, J. E. Bates, J. Sun, and J. P. Perdew, "Benchmark tests of a strongly constrained semilocal functional with a long-range dispersion correction," Phys. Rev. B, vol. 94, p. 115144, Sept. 2016.

[28] Y. Zhao and D. G. Truhlar, "A new local density functional for main-group thermochemistry, transition metal bonding, thermochemical kinetics, and noncovalent interactions," The Journal of Chemical Physics, vol. 125, p. 194101, Nov. 2006.

[29] L. Goerigk and S. Grimme, "A thorough benchmark of density functional methods for general main group thermochemistry, kinetics, and noncovalent interactions," Phys. Chem. Chem. Phys., vol. 13, no. 14, p. 6670, 2011. 
[30] N. Mardirossian and M. Head-Gordon, "SB97M-V: A combinatorially optimized, range-separated hybrid, meta-GGA density functional with VV10 nonlocal correlation," The Journal of Chemical Physics, vol. 144, p. 214110, June 2016.

[31] A. D. Becke, "A new mixing of Hartree-Fock and local density-functional theories," The Journal of Chemical Physics, vol. 98, pp. 1372-1377, Jan. 1993.

[32] P. J. Stephens, F. J. Devlin, C. F. Chabalowski, and M. J. Frisch, "Ab initio calculation of vibrational absorption and circular dichroism spectra using density functional force fields," J. Phys. Chem., vol. 98, pp. 11623-11627, Nov. 1994.

[33] N. Mardirossian and M. Head-Gordon, " $\omega$ B97X-V: A 10-parameter, range-separated hybrid, generalized gradient approximation density functional with nonlocal correlation, designed by a survivalof-the-fittest strategy," Phys. Chem. Chem. Phys., vol. 16, no. 21, p. 9904, 2014.

[34] Y. Zhao, N. E. Schultz, and D. G. Truhlar, "Design of density functionals by combining the method of constraint satisfaction with parametrization for thermochemistry, thermochemical kinetics, and noncovalent interactions," J. Chem. Theory Comput., vol. 2, pp. 364-382, Mar. 2006.

[35] K. Pernal, R. Podeszwa, K. Patkowski, and K. Szalewicz, "Dispersionless density functional theory," Phys. Rev. Lett., vol. 103, p. 263201, Dec. 2009.

[36] R. Podeszwa, K. Pernal, K. Patkowski, and K. Szalewicz, "Extension of the Hartree-Fock plus dispersion method by first-order correlation effects," J. Phys. Chem. Lett., vol. 1, pp. 550-555, Jan. 2010.

[37] S. Kozuch and J. M. L. Martin, "Spin-component-scaled double hybrids: An extensive search for the best fifth-rung functionals blending DFT and perturbation theory," J. Comput. Chem., pp. 2327-2344, July 2013.

[38] S. Grimme, "Semiempirical hybrid density functional with perturbative second-order correlation," Journal of Chemical Physics, vol. 124, no. 3, pp. 034108-034108, 2006. 
[39] L. Goerigk and S. Grimme, "Efficient and accurate double-hybrid-meta-GGA density functionals - Evaluation with the extended GMTKN30 database for general main group thermochemistry, kinetics, and noncovalent interactions," J. Chem. Theory Comput., vol. 7, pp. 291-309, Feb. 2011.

[40] W. Hujo and S. Grimme, "Performance of the van der waals density functional VV10 and (hybrid)GGA variants for thermochemistry and noncovalent interactions," Journal of Chemical Theory and Computation, vol. 7, no. 12, pp. 3866-3871, 2011.

[41] F. Yu, "Spin-component-scaled double-hybrid density functionals with nonlocal van der Waals correlations for noncovalent interactions," J. Chem. Theory Comput., vol. 10, pp. 4400-4407, Oct. 2014 .

[42] E. Brémond and C. Adamo, "Seeking for parameter-free double-hybrid functionals: The PBE0-DH model," The Journal of Chemical Physics, vol. 135, p. 024106, July 2011.

[43] D. Bousquet, E. Brémond, J. C. Sancho-García, I. Ciofini, and C. Adamo, "Non-parametrized functionals with empirical dispersion corrections: A happy match?," Theor Chem Acc, vol. 134, p. 1602, Jan. 2015.

[44] S. Lehtola, "Fully numerical Hartree-Fock and density functional calculations. II. Diatomic molecules," Int J Quantum Chem, vol. 119, Oct. 2019.

[45] T. Gould, "Diet GMTKN55' offers accelerated benchmarking through a representative subset approach," Phys. Chem. Chem. Phys., vol. 20, no. 44, pp. 27735-27739, 2018.

[46] P. Kraus, D. A. Obenchain, and I. Frank, "Benchmark-quality semiexperimental structural parameters of van der Waals complexes," J. Phys. Chem. A, vol. 122, pp. 1077-1087, Feb. 2018.

[47] P. Kraus, "Non-covalent dimers and trimers (NCDT) database version 2.0," Feb. 2020.

[48] A. J. C. Varandas, "CBS extrapolation of Hartree-Fock energy: Pople and Dunning basis sets hand-to-hand on the endeavour," Phys. Chem. Chem. Phys., vol. 21, no. 15, pp. 8022-8034, 2019.

[49] S. Lehtola, "A review on non-relativistic, fully numerical electronic structure calculations on atoms and diatomic molecules," Int J Quantum Chem, vol. 119, p. e25968, Oct. 2019. 
[50] K. N. Kirschner, D. Reith, and W. Heiden, "The performance of Dunning, Jensen, and Karlsruhe basis sets on computing relative energies and geometries," Soft Materials, pp. 1-15, Feb. 2020.

[51] K. Jankowski, P. Malinowski, and M. Polasik, "Second-order correlation energies for $\mathrm{F}^{1-}, \mathrm{Na}^{1+}$, $\mathrm{Mg}^{2+}$, and $\mathrm{Ar}^{8+}: \mathrm{Z}$ dependence of irreducible-pair energies," Phys. Rev. A, vol. 22, pp. 51-60, July 1980.

[52] J. R. Flores, "Hierarchic computation of atomic correlation energies using a p-version finite element method," Chemical Physics Letters, vol. 195, pp. 377-382, July 1992.

[53] J. R. Flores and P. Redondo, "Accurate second-order correlation energies for Mg and Ar," Int. J. Quantum Chem., vol. 45, no. 6, pp. 563-572, 1993.

[54] J. R. Flores, "New benchmarks for the second-order correlation energies of Ne and Ar through the finite element MP2 method," Int. J. Quantum Chem., vol. 108, pp. 2172-2177, May 2008.

[55] J. Noga, P. Valiron, and W. Klopper, "The accuracy of atomization energies from explicitly correlated coupled-cluster calculations," The Journal of Chemical Physics, vol. 115, pp. 2022-2032, Aug. 2001.

[56] A. Najibi and L. Goerigk, "The nonlocal kernel in van der Waals density functionals as an additive correction: An extensive analysis with special emphasis on the B97M-V and $\omega$ B97M-V approaches," J. Chem. Theory Comput., vol. 14, pp. 5725-5738, Nov. 2018.

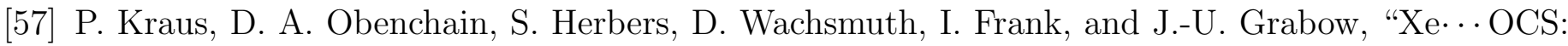
Relatively straightforward?," Phys. Chem. Chem. Phys., vol. 22, no. 10, pp. 5615-5624, 2020. 